Supporting information

\title{
Metabonomics Approach to Assessing the Metabolism Variation and Endo-exogenous Metabolic Interaction of Ginsenosides in Cold Stress Rats
}

\author{
Zhihao Zhang ${ }^{1, \dagger}$, Xiaoyan Wang ${ }^{1 * \dagger}$, Jingcheng Wang ${ }^{\dagger}$, Zhiying Jia ${ }^{\dagger}$, Yumin Liu ${ }^{i}$, Xie Xie ${ }^{\dagger}$, Chongchong \\ Wang ${ }^{\dagger}$ \\ ${ }^{\dagger}$ Ministry of Education Key Laboratory of Systems Biomedicine, Shanghai Center for Systems Biomedicine and \\ 'Instrumental Analysis Center of SJTU , Shanghai Jiao Tong University, Shanghai, 200240, P. R. China
}

\section{Table of Contents}

Method validation of LC-MS

Table S1. Differentially expressed urine metabolites between pre-exposure and post-exposure to cold stress in TG group in positive ion mode.

Figure S1. Effects of total ginsenosides on urinary metabolic variation. PLS-DA score plot of GC/MS spectral data from control group and TG group at week 2 (A). Effects of cold stress on variation of 18 ginsenosides concentration in urine. PLS-DA score plot of LC/MS spectral data from urine samples in TG group at these two states (pre-exposure to cold and postexposure to cold) (B).

Figure S2. (A) Hierarchical clustering of differential ginsenosides. Correlation analysis of the 8 differential ginsenosides in the TG group between the pre- and post-cold stress exposure. (B) Correlation of alterations of 3 significantly altered ginsenosides (between pre- and post- exposure to cold, $n=7$ ) with the alterations of differentially expressed metabolites (between pre- and post- exposure to cold, $\mathrm{n}=7$ ).

\footnotetext{
${ }^{*}$ To whom correspondence should be addressed:

Xiaoyan Wang at Ministry of Education Key Laboratory of Systems Biomedicine, Shanghai Center for Systems Biomedicine, Shanghai Jiao Tong University, Shanghai 200240, China. Phone: 86-21-34207343;Fax: 86-21-34206059;

E-mail: cathywxy@sjtu.edu.cn

Wei Jia at at Ministry of Education Key Laboratory of Systems Biomedicine, Shanghai Center for Systems Biomedicine, Shanghai Jiao Tong University, Shanghai 200240, China. Phone: 86-21-34207343;Fax: 86-21-34206059; E-mail: weijia@sjtu.edu.cn

${ }^{1}$ These authors contributed equally to this work.
} 


\section{Method validation of LC-MS}

The regression coefficient $\left(\mathrm{r}^{2}\right)$ of each ginsenoside's linear regression equation are higher than 0.99 . Recovery, intraday and interday precision were evaluated by analyzing triplicate samples of three concentrations of rat urine. The repeatability was validated by the reduplicate analysis of three injections of the same quality control samples. Recovery, intraday precision, interday precision and repeatability were expressed as R.S.D. The intraday precision was less than $11 \%$. The interday precision, recovery and repeatability were less than $12 \%$. 
Table S1.

\begin{tabular}{|c|c|c|c|c|}
\hline \multirow{2}{*}{ Metabolites } & \multicolumn{4}{|c|}{ post-cold stress exposure vs pre-cold stress exposure } \\
\hline & $\mathrm{VIP}^{\mathrm{a}}$ & Fold Change $\mathrm{e}^{\mathrm{b}}$ & $\mathrm{p}(\mathrm{T} \text {-test })^{\mathrm{c}}$ & p (Mann-Whitney) ${ }^{\mathrm{d}}$ \\
\hline Leucine & 2.02 & 0.15 & $1.49 \mathrm{E}-03$ & $1.51 \mathrm{E}-02$ \\
\hline Butanedioic acid & 1.76 & 2.40 & $9.22 \mathrm{E}-03$ & $1.09 \mathrm{E}-02$ \\
\hline Picolinoylglycine & 1.73 & 0.36 & $1.09 \mathrm{E}-02$ & $1.51 \mathrm{E}-02$ \\
\hline Benzaldehyde & 1.67 & 4.06 & $1.45 \mathrm{E}-02$ & 2.79E-02 \\
\hline Quinolinic acid & 1.64 & 3.01 & $1.69 \mathrm{E}-02$ & $6.41 \mathrm{E}-02$ \\
\hline Isopentanol & 1.53 & 2.48 & $2.90 \mathrm{E}-02$ & $3.72 \mathrm{E}-02$ \\
\hline Glutaric acid & 1.52 & 4.25 & 3.04E-02 & $1.09 \mathrm{E}-02$ \\
\hline 3-Hydroxyisovaleric acid & 1.44 & 1.82 & 4.17E-02 & 2.79E-02 \\
\hline Isoleucine & 1.44 & 0.20 & 4.26E-02 & 4.91E-02 \\
\hline Hexanedioic acid & 1.44 & 2.06 & 4.30E-02 & $1.05 \mathrm{E}-01$ \\
\hline Butanedioic acid & 1.43 & 0.26 & 4.44E-02 & 2.79E-02 \\
\hline Ethanedioic acid & 1.42 & 0.54 & 4.49E-02 & 4.91E-02 \\
\hline Hydroxycinnamic acid & 1.42 & 13.01 & 4.63E-02 & $6.41 \mathrm{E}-02$ \\
\hline Phenylalanine & 1.39 & 0.47 & 5.09E-02 & 4.91E-02 \\
\hline \multicolumn{5}{|c|}{${ }^{a}$ Variable importance in the projection (VIP) was obtained from the PLS-DA model. ${ }^{b} \mathrm{Fold}$ change was calculated as a binary logarithm of the average mass response } \\
\hline \multicolumn{5}{|c|}{ (normalized peak area) ratio between post-cold stress exposure vs pre-cold stress exposure, where a positive value means that the average mass response of post-cold } \\
\hline \multicolumn{5}{|c|}{ stress exposure is larger than pre-cold stress exposure. ${ }^{\mathrm{c}}$ The $p$ value was calculated from Student's $\mathrm{t}$ test. ${ }^{\mathrm{d}}$ The $p$ value was calculated from nonparametric test } \\
\hline Mann-Whitney U test. & & & & \\
\hline
\end{tabular}


Figure S1
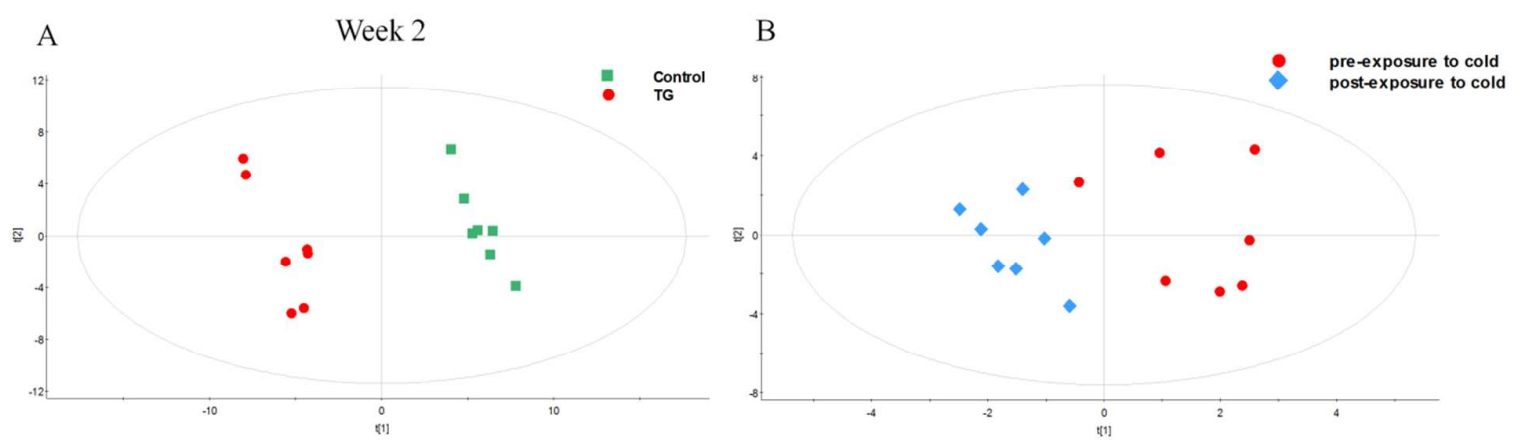
Figure S2

A

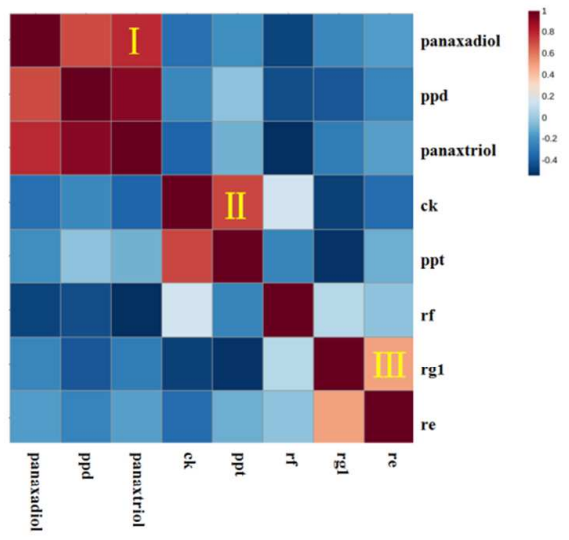

B

Rg1 Re PPD

\begin{tabular}{|l|l|}
\hline Leucine \\
\hline Butanedioic acid \\
\hline Picolinoylglycine \\
\hline Benzaldehyde \\
Quinolinic acid \\
\hline Isopentanol \\
Glutaric acid \\
\hline 3-Hydroxyisovaleric acid \\
\hline Isoleucine \\
Hexanedioic acid \\
Butanedioic acid \\
Ethanedioic acid \\
Hydroxycinnamic acid \\
Phenylalanine
\end{tabular}

$\begin{array}{ll}0.8 & 0.8\end{array}$

Correlation coefficient 